\title{
Intranasal erythropoietin ameliorates neurological function impairments and neural pathology in mice with chronic alcoholism by regulating autophagy-related $\mathrm{Nrf} 2$ degradation
}

\author{
XUEDAN NIE ${ }^{1}$, WENBO WANG ${ }^{2}$, QIN WANG $^{3}$, DAN ZHU $^{3}$ and HONGSHAN SONG ${ }^{4}$ \\ ${ }^{1}$ Department of Neurology, The Second Affiliated Hospital of Harbin Medical University, Harbin, Heilongjiang 150001; \\ ${ }^{2}$ Intensive Care Unit, The Fifth Affiliated Hospital of Qiqihar Medical University (Daqing Longnan Hospital), Daqing, \\ Heilongjiang 163453; ${ }^{3}$ Department of Nephrology, The Second Affiliated Hospital of Harbin Medical University, \\ Harbin, Heilongjiang 150001; ${ }^{4}$ Department of Neurology, The Fifth Affiliated Hospital of \\ Qiqihar Medical University (Daqing Longnan Hospital), Daqing, Heilongjiang 163453, P.R. China
}

Received April 5, 2018; Accepted November 1, 2018

DOI: $10.3892 / \mathrm{mmr} .2018 .9706$

\begin{abstract}
The neurological disorders and neural pathology brought about by chronic alcoholism are difficult to be reversed. Increasing evidence highlights the protective roles of erythropoietin (EPO) in neurodegenerative diseases and injuries of the central nervous system. In the present study, we investigated the therapeutic effects of EPO on the neurological function deficits and neural pathology caused by chronic alcoholism and the regulatory mechanisms. Using the canonical mouse model of chronic alcohol exposure designed to mimic the repeated cycles of heavy abuse typical of chronic alcoholism, it was found that EPO delivered via intranasal route effectively restored the alcohol-impaired motor cooperation in rotarod and beam walk tests, reversed alcoholic cognitive and emotional alterations in the novel location recognition task and open-filed test, and rescued alcohol-disrupted nervous conduction in the somatosensory-evoked potential (SSEP) test. Consistently, the intranasally administered EPO promoted the remyelination
\end{abstract}

Correspondence to: Dr Hongshan Song, Department of Neurology, The Fifth Affiliated Hospital of Qiqihar Medical University (Daqing Longnan Hospital), 35 Aiguo Road, Daqing, Heilongjiang 163453, P.R. China

E-mail: drsong_longnanh@163.com

Abbreviations: ATRA, all-trans-retinoic acid; CNS, central nervous system; CAE, chronic alcohol exposure; CIE, chronic intermittent ethanol; EEG, electroencephalography; EPOR, erythropoietin receptor; EPO, erythropoietin; HO1, heme oxygenase 1; HRP, horseradish peroxidase; KEAP1, Kelch-like ECH-associated protein 1; MBP, myelin basic protein; NQO1, NADP, H quinone oxidoreductase; Nrf2, nuclear factor, erythroid 2-like 2; rhEPO, recombinant human erythropoietin; SSEP, somatosensory-evoked potential

Key words: erythropoietin, chronic alcoholism, neurological function deficits, Nrf2, autophagy and synapse formation in chronic alcohol-affected neocortex and hippocampus as evidenced by immunofluorescence staining and transmission electron microscopy. Additionally, we discovered that the exogenous rhEPO, which entered the cerebrum through intranasal route, activated the erythropoietin receptor (EPOR) and the downstream ERKs and PI3K/AKT signaling, and suppressed autophagy-related degradation of nuclear factor, erythroid 2-like 2 (Nrf2). Furthermore, the intranasal EPO-exerted neuroprotection was almost abolished when the specific Nrf2 antagonist ATRA was administered intraperitoneally prior to intranasal EPO treatment. Collectively, our data demonstrated the repairing potential of EPO for the neurological disorders and neural pathology caused by chronic alcoholism, and identified the Nrf2 activity as the key mechanism mediating the protective effects of EPO.

\section{Introduction}

As estimated by the World Health Organization, 280 million individuals over 15 years of age (4.1\% of the population) are engaged in repeated excessive episodic alcohol consumption and are affected by chronic alcoholism (1). Patients with chronic alcoholism (i.e., Wernicke's encephalopathy) frequently exhibit motor dysfunction, memory and cognitive impairments and mental disorders $(2,3)$. Of note, some disorders, such as memory deficits in chronic alcoholism, are generally irreversible and inclined to progress into alcoholic dementia (4). The neurological disorders of chronic alcoholism are closely associated with neurodegeneration, synapse loss and myelin damage probably resulting from sustained alcohol exposure-induced neurotrophin exhaustion, oxidative stress and neuroinflammation, in the prefrontal cortex and limbic system structures (hippocampus and amygdala) that are particularly vulnerable to the neurotoxic effects of alcohol (1,4-6). To date, there is no effective therapy to reverse the permanent deficits of chronic alcoholism.

Erythropoietin (EPO) is a $30-\mathrm{kDa}$, hypoxia-sensitive glycoprotein with erythropoiesis-promoting properties. The EPO receptor (EPOR) via which EPO functions is found to 
be expressed ubiquitously in the mammalian central nervous system (CNS). Recent evidence highlights the protective roles of EPO in neurodegenerative diseases (7-9). EPO treatment was found to reduce motor neuron degeneration and delay the motor deterioration onset in amyotrophic lateral sclerosis mouse models $(7,8)$, and to alleviate learning and memory deficits and attenuate $\mathrm{A} \beta$-induced neurotoxicity in an Alzheimer's disease mouse model (9). In addition, data from preclinical studies have demonstrated that EPO also contributes to the histological and functional recoveries from various traumatic and ischemic injuries of CNS (10-12). Mechanistically, diverse signaling pathways downstream of EPO/EPOR (i.e., ERKs and PI3K/AKT signaling) have been implicated in the neuroprotection of EPO $(12,13)$. However, the exact impacts of EPO on chronic alcoholism-related neuropathology and neurological dysfunctions remain to be elucidated.

Nuclear factor, erythroid 2-like 2 (Nrf2) is the master transcription factor orchestrating antioxidant and detoxification responses (14-16). Under homeostatic conditions, Nrf2 is sequestered by Kelch-like ECH-associated protein 1 (KEAP1) in the cytoplasm and is consequently degraded by the proteasome. When cells undergo stress, Nrf2 dissociates from KEAP1 and translocates into the nucleus to activate the expression of its downstream target genes such as $\mathrm{NAD}(\mathrm{P}) \mathrm{H}$ quinone oxidoreductase (NQO1), heme oxygenase 1 (HO1) and glutamate-cysteine ligases. Intriguingly, autophagy-degraded p62 can regulate Nrf2 activity positively by inactivating KEAP1, which has been observed during the neuroectoderm commitment of embryonic stem cell and induced inflammatory tolerance of macrophages $(15,17)$. As reported recently, the elevated Nrf2 activity can also be evoked by EPO-stimulated ERKs and PI3K/AKT pathways, and Nrf2 can mediate the neuroprotective effects of EPO (18-20). Nevertheless, although oxidative stress plays a critical role in chronic alcoholism-related injuries (21-23), whether Nrf2 activity is involved in the autonomous and non-autonomous repair of alcoholic brain damage remains elusive.

In this study, the therapeutic effects of EPO on chronic alcoholism-related neural pathology and brain disorders and as well as the involvement of Nrf2 activity were investigated on mice subjected to chronic alcohol exposure.

\section{Materials and methods}

Animals and experimental designs. Male C57BL/6J mice at 12 weeks of age weighing 22-25 g were purchased from the Model Animal Research Center of Nanjing University and maintained on a 12-h light/dark cycle with controlled temperature $\left(22-23^{\circ} \mathrm{C}\right)$ and humidity (45-55\%). For all repertoires of experiments, $\mathrm{C} 57 \mathrm{BL} / 6 \mathrm{~J}$ mice were randomly assigned to the Control, Chronic Alcohol Exposure (CAE), $\mathrm{CAE}+$ recombinant human erythropoietin (rhEPO) and $\mathrm{CAE}+$ rhEPO+all-trans-retinoic acid (ATRA) groups on average ( 8 mice/group). The CAE, CAE+rhEPO and $\mathrm{CAE}+\mathrm{rhEPO}+\mathrm{ATRA}$ groups were subjected to alcohol exposure for 4 weeks. Successively, the CAE+rhEPO and $\mathrm{CAE}+\mathrm{rhEPO}+\mathrm{ATRA}$ groups were treated with rhEPO in saline [100 IU/ml, 500 IU/kg body weight; Roche Diagnostics (Shanghai) Ltd., China] intranasally every 12 h for 4 weeks, while the CAE group was treated only with an equal amount of vehicle. Simultaneously, the CAE+rhEPO+ATRA group received intraperitoneal administration of ATRA in saline (2 mg/ml, 20 mg/kg; Sigma-Aldrich; Merck KGaA, Darmstadt, Germany) 30 min before intranasal rhEPO treatment for 4 weeks, while other groups received merely an equal amount of vehicle. Animals in the Control group underwent all procedures except chronic alcohol exposure and medication. For neural function analyses, neurological deficits were assessed by motor cooperation, cognition and nerve conduction tests $12 \mathrm{~h}$ after rhEPO administration for the last time. For the following histopathological analyses, animals were decapitated and the brain specimens were dissected and subjected to immunostaining and electron microscopy. For the independent mechanism study, the homogenate of brain tissue of animals was subjected to western blotting analyses for neural damage repair-related key molecules 30 min immediately after the drug treatment for the last time. All experimental procedures on animals were approved by the Ethics Committee of Harbin Medical University and the Ethics Committee of Qiqihar Medical University, and followed the principles of medical ethics.

Alcohol exposure. Chronic alcohol exposure was achieved via a chronic intermittent ethanol (CIE) vapor inhalation procedure previously described for C57BL/6J mice (24). Briefly, mice were placed in standard mouse cages in Plexiglas vapor chambers $(60 \times 36 \times 60 \mathrm{~cm}$, PlasLabs, up to six cages per chamber) and exposed to continuous fresh air-mixed vaporized which delivered 19-22 $\mathrm{mg}$ of alcohol per liter of air at a rate of $\sim 101 / \mathrm{min}$ and produced blood alcohol levels of $175 \pm 25 \mathrm{mg} / \mathrm{dl}$ as designed. The blood alcohol level was verified weekly via blood samples taken from dedicated 'sentinel' mice exposed to alcohol simultaneously with the test mice. To initiate intoxication and stabilize blood alcohol levels, the alcohol groups received intraperitoneal injections of $1 \mathrm{mmol} / \mathrm{kg}$ body weight of the alcohol dehydrogenase inhibitor pyrazole (Sigma-Aldrich; Merck KGaA) combined with $1.5 \mathrm{~g} / \mathrm{kg}$ body weight of $20 \%$ alcohol (v/v), in a volume of $10 \mathrm{ml} / \mathrm{kg}$ body weight, before placement in the chambers. Exposure lasted for $16 \mathrm{~h}$ each day (in at 17:00 h, $2 \mathrm{~h}$ before the start of the 12-h circadian dark phase, out at 09:00 h), followed by an 8 -h withdrawal. There were 4 consecutive days of intermittent exposure (Monday through Thursday) and then an 80-h withdrawal (Friday through Monday). This complete weekly cycle was repeated four times. Controls received an injection of $1 \mathrm{mmol}$ per $\mathrm{kg}$ of body weight pyrazole in saline to control for this treatment, before placement in dedicated air chambers (located adjacent to the alcohol chambers), which received vaporized air at the same exchange rate as the alcohol chambers.

Intranasal administration of rhEPO. The intranasal administration was performed as reported previously (25). In brief, recombinant human (rh) EPO [Roche Diagnostics (Shanghai) Ltd.] was prepared using sterile $0.45 \%$ normal saline solution at a concentration of $100 \mathrm{IU} / \mathrm{ml}$ and stored at $-20^{\circ} \mathrm{C}$. Each mouse was held ventral side up, a small-modified 27-French catheter was inserted into either nares, and saline or rhEPO was slowly administered intranasally at no more than $5 \mu \mathrm{l}$ increments 5-10 min apart for a total of $500 \mathrm{IU} / \mathrm{kg}$ body weight. 
The mouse was held for 1-2 min to ensure absorption. Drug or saline was administered every $12 \mathrm{~h}$ beginning immediately after the 4-week alcohol exposure.

Motor cooperation tests. Motor cooperation of mice was assessed by rotarod and beam walking assays. For the motor tests, the training began 3 days before alcohol exposure and the tests were conducted 1 week after treatment. The rotarod assay was conducted using a ROTO-ROD series 8 (IITC Inc./Life Science, Inc., Woodland Hills, CA, USA) which could accelerate rotational speed from 4 to $40 \mathrm{rpm}$ over $120 \mathrm{sec}$. The mean latency of mice to fall off the rotarod in the three trials was recorded as measurement. For the beam walking assay, the time mice spent in crossing a $1-\mathrm{m}$ long and $3-\mathrm{cm}$ thick beam was calculated for analysis.

Cognition and activity tests. Novel location recognition and open-field tests were used to evaluate the spatial hippocampal-dependent memory, activity and anxiety. For the novel location recognition test, each mouse was allowed to explore the empty arena $(50-\mathrm{cm}$ diameter) for $10 \mathrm{~min}$ on the 1 st day for familiarization. In this arena, two identical objects were placed in the middle on the 2 nd day for acquisition, and in the same arena, one of the two objects was moved to a novel location on the 3rd day for the test. The exploration time for the two objects in the acquisition and test phase was recorded. The percentage of the time mice spent exploring the displaced object in the total exploration time for both objects was defined as the preference index. For the open-field test, mice were placed into a chamber measuring $50 \times 50 \times 38 \mathrm{~cm}$ for $60 \mathrm{~min}$. The total number of forelimb rears, and the ratio of time spent in the center to the time spent in the corners were recorded as measurements.

Nerve conduction test. Somatosensory-evoked potential (SSEP) was monitored to assess cortical synaptic transmission and nerve conduction. To generate forepaw stimulation, 2 metal pins were inserted into the left forepaw and a $1-5 \mathrm{~mA}$ 1 or $2 \mathrm{msec}$ electrical pulse was delivered by constant current stimulus output using the RM6240C Multi-channel Bioelectric Signal Acquisition System (Chengdu Instrument Factory, Sichuan, China). For electroencephalography (EEG) recording, a Teflon-coated silver chloride wire $(125 \mu \mathrm{m})$ with an exposed tip $(1 \mathrm{~mm})$ as the EEG electrode was placed on the surface of the primary somatosensory cortex corresponding to the left forelimb within the agarose, and the reference electrode was placed on the nasal bone under the skin. The cortical signal was amplified, filtered and recorded using the RM6240C Multi-channel Bioelectric Signal Acquisition System. The onset latency and the amplitude of the large primary cortical wave were determined.

Immunofluorescence staining. Mice were anesthetized with isoflurane and carbon dioxide as dictated by ethical guidelines and decapitated. The brains were dissected rapidly, fixed overnight in $4 \%$ paraformaldehyde and processed for paraffin embedding. For immunofluorescence staining, $4-\mu \mathrm{m}$ paraffin sections were prepared. After deparaffinization and rehydration according to standard procedures, sections underwent antigen retrieval in citrate buffer ( $\mathrm{pH}$ 6.0) using a microwave oven $(750 \mathrm{~W})$. Non-specific antibody binding was blocked with $5 \%$ bovine serum albumin (BSA), $0.2 \%$ Triton X-100 in PBS for $1 \mathrm{~h}$ at room temperature. Subsequently, sections underwent incubation with primary antibodies [rabbit polyclonal anti-human/mouse/rat synaptophysin (IgG; 1:100; catalog no. SAB4502906; Sigma-Aldrich; Merck KGaA) and rabbit polyclonal anti-human/mouse/rat myelin basic protein (MBP) (IgG; 1:100; catalog no. 78896; Cell Signaling Technology, Inc., Danvers, MA, USA)] diluted in 2\% BSA, $0.1 \%$ Triton in PBS overnight at $4^{\circ} \mathrm{C}$. Following the washing out of the unbound antibodies by PBS, the sections underwent incubation with the secondary antibody [goat polyclonal anti-rabbit IgGs conjugated to Alexa Fluor ${ }^{\circledR} 488$ (1:1,000; catalog no. A32723; Invitrogen, Thermo Fisher Scientific, Inc., Waltham, MA, USA)] diluted in $2 \%$ BSA, $0.1 \%$ Triton in PBS in dark for $1 \mathrm{~h}$ at room temperature, and counterstained with DAPI ( $1 \mu \mathrm{g} / \mathrm{ml}$; Sigma-Aldrich; Merck KGaA) in methanol for nuclear staining. The stained sections were mounted with anti-fade reagent (Invitrogen, Thermo Fisher Scientific) and photographed under a BX51 fluorescence microscope (Olympus, Tokyo, Japan). The fluorescence intensity was measured using Image-Pro Plus 6.0 (Media Cybernetics, Inc., Rockville, MD, USA).

Transmission electron microscopy. Samples of dissected brain were fixed in $0.1 \mathrm{M}$ sodium-cacodylate-buffered mixture (5\% glutaraldehyde and 4\% paraformaldehyde), post-fixed in $1 \%$ osmium tetroxide and $1.5 \%$ potassium ferrocyanide, dehydrated and embedded in epoxy resin. Ultrathin sections (80 nm) were then cut, collected on copper grids, stained with lead citrate and uranyl acetate for contrast and examined under H-7650 electron microscope (Hitachi, Osaka, Japan) equipped with a MegaView III CCD camera (Soft Imaging System, Lakewood, CO, USA). The synapse densities in the captured images were quantified using Adobe Photoshop Creative Cloud.

Western blotting. The dissected brain tissue was homogenized and lysed using RIPA lysis buffer (Beyotime, Zhejiang, China) supplemented with protease and phosphatase inhibitors (Complete and Phosphostop, Roche, Upper Bavaria, Germany) for $30 \mathrm{~min}$ on mice, and centrifugation at $12,000 \mathrm{x} \mathrm{g}$ for $20 \mathrm{~min}$ at $4^{\circ} \mathrm{C}$. The supernatant was harvested, and the protein concentration was determined using the BCA kit (Beyotime). SDS sample buffer containing $\beta$-mercaptoethanol was added to the protein lyses. Totally $30 \mu \mathrm{g}$ protein was loaded per lane, resolved by $8-12 \%$ polyacrylamide gels in a Mini-PROTEAN ${ }^{\circledR}$ Tetra Vertical Electrophoresis Cell (Bio-Rad Laboratories, Inc., Hercules, CA, USA), electroblotted onto polyvinylidene fluoride membranes (Merck Millipore, Darmstadt, Germany) in a Mini Trans-Blot ${ }^{\circledR}$ Module (Bio-Rad Laboratories), blocked with $5 \%$ skimmed milk/TBST, and incubated with the primary antibodies diluted in the blocking buffer overnight at $4^{\circ} \mathrm{C}$. The following primary antibodies were used: mouse monoclonal anti-human hEPO (catalog no. SAB5300475; Sigma-Aldrich; Merck KGaA), rabbit polyclonal anti-human/mouse/rat p-EPOR (catalog no. SAB4301492; Sigma-Aldrich; Merck KGaA), rabbit polyclonal anti-human/mouse/rat p-ERK1/2 (catalog no. E7028; Sigma-Aldrich; Merck KGaA), rabbit polyclonal anti-human/mouse/rat p-AKT (catalog 
no. SAB4301497; Sigma-Aldrich; Merck KGaA), rabbit polyclonal anti-human/mouse/rat Nrf2 (catalog no. SAB4501984; Sigma-Aldrich; Merck KGaA), rabbit polyclonal anti-human/mouse/rat KEAP1 (catalog no. AV34727; Sigma-Aldrich; Merck KGaA), rabbit polyclonal anti-human/mouse/ratNQO1 (catalogno.N5288;Sigma-Aldrich; Merck KGaA), rabbit polyclonal anti-human/mouse/rat p62 (catalog no. P0067; Sigma-Aldrich; Merck KGaA), rabbit polyclonal anti-human/mouse/rat LC3B (catalog no. 3868; Cell Signaling Technology, Inc.) and rabbit polyclonal anti-human/mouse/rat $\beta$-actin (catalog no. SAB2100037; Sigma-Aldrich; Merck KGaA) (IgGs; 1:1,000). $\beta$-actin expression was used as a loading control. After the washout of the unbound primary antibodies, the membranes underwent incubation with the secondary antibodies [horseradish peroxidase (HRP)-conjugated goat polyclonal anti-mouse IgG (1:5,000; catalog no. 31430; Invitrogen; Thermo Fisher Scientific, Inc.) or HRP-conjugated goat polyclonal anti-rabbit $\operatorname{IgG}(1: 5,000$; catalog no. 31460; Invitrogen; Thermo Fisher Scientific, Inc.)] for $1 \mathrm{~h}$ at room temperature. Protein blots were visualized using a ChemiDoc ${ }^{\mathrm{TM}}$ Imaging System (Bio-Rad), and quantified using Image-Pro Plus 6.0.

Data processing and statistics. Data are expressed as the mean \pm standard error of the mean (SEM). Statistical analyses were performed using GraphPad Prism 7.0 software (GraphPad Software, Inc., La Jolla, CA, USA). Statistical differences were determined by one-way analysis of variance (ANOVA) followed by Bonferroni's multiple comparisons tests (more than two experimental conditions) and Wilcoxon signed-rank test (when compared with $50 \%$ chance level). $\mathrm{P}<0.05$ was considered statistically significant.

\section{Results}

Intranasal rhEPO reverses the motor function deficits in mice with chronic alcoholism. To evaluate the impacts of rhEPO administered via intranasal route on the motor function of mice exposed to alcohol for 4 weeks, rotarod and beam walk tests were conducted after a 4-week rhEPO treatment. In the rotarod test (Fig. 1A), mice exposed to sustained alcohol in the CAE group showed dramatically shortened time remaining on the rotarod when compared with mice in the Control group (CAE vs. Control, $\mathrm{P}<0.001)$, whereas alcohol-exposed mice receiving intranasal rhEPO administration in the CAE+rhEPO group presented with significantly longer time maintaining on the rotarod $(\mathrm{CAE}+\mathrm{rhEPO}$ vs. $\mathrm{CAE}, \mathrm{P}<0.05)$, up to the level of the Control group (CAE+rhEPO vs. Control, $\mathrm{P}>0.05$ ). When challenged on a $1-\mathrm{cm}$ beam, mice in the CAE group needed more time to cross the beam than those in the Control group (CAE vs. Control, $\mathrm{P}<0.001$; Fig. 1B). In contrast, mice in the CAE+rhEPO group spent less time crossing the beam $(\mathrm{CAE}+\mathrm{rhEPO}$ vs. $\mathrm{CAE}, \mathrm{P}<0.05)$ and regained similar beam-crossing capacity to that of mice in the Control group (CAE+rhEPO vs. Control, $\mathrm{P}>0.05$ ). Data for the motor tests indicated that intranasal rhEPO treatment could promote recovery from chronic alcoholism-related motor deficits.

Intranasal rhEPO alleviates the cognitive disorders in mice with chronic alcoholism. To examine the effects of intranasal
rhEPO on cognition and anxiety following chronic alcoholism, mice in each group were tested in object location and open-field tasks post rhEPO treatment (4 weeks post alcohol exposure). During the acquisition phase in the novel location recognition task (Fig. 1C), mice in all groups spent the same amount of time exploring the objects. During memory testing $24 \mathrm{~h}$ later (Fig. 1D), mice in the Control and CAE+rhEPO groups similarly explored preferentially the object that had been moved to a novel location (CAE+rhEPO vs. Control, $\mathrm{P}>0.05$; Control and CAE+rhEPO vs. chance level 50\%, $\mathrm{P}<0.01$ ), but mice in the CAE group showed no exploratory preference for the displaced object (CAE vs. Control, $\mathrm{P}<0.001$; CAE vs. CAE+rhEPO, P<0.01; CAE vs. chance level 50\%, $\mathrm{P}>0.05$ ), indicating that the impaired discriminative memory of mice with chronic alcoholism for the novel and familiar location could be rescued by intranasal rhEPO. During the open-field test (Fig. 1E and F), alcohol-exposed mice without drug treatment had sharply diminished forelimb rears (CAE vs. Control, $\mathrm{P}<0.001$ ), decreased time spent in the center and consequentially increased time spent in the corners of the open field (CAE vs. Control, $\mathrm{P}<0.001$ ) compared to the healthy control mice, indicative of reduced exploration and aggravated anxiety. However, intranasal rhEPO administration remarkably restored vertical exploration activity (CAE+rhEPO vs. CAE, $\mathrm{P}<0.001$; CAE+rhEPO vs. Control, $\mathrm{P}>0.05$ ), increased center exploration and relieved anxiety (CAE+rhEPO vs. CAE, $\mathrm{P}<0.001$; $\mathrm{CAE}+$ rhEPO vs. Control, $\mathrm{P}>0.05$ ) in mice exposed to alcohol for 4 weeks. These findings in cognitive and behavioral tests suggested that intranasal rhEPO treatment could ameliorate the memory, learning and anxiety disorders in mice with chronic alcoholism.

Intranasal rhEPO rescues the nervous conduction impairments in mice with chronic alcoholism. To further determine the effects of intranasal rhEPO on nervous conduction after chronic alcohol exposure, somatosensory-evoked potential (SSEP) analysis was performed. In this study, the amplitude and latency of SSEP were selected as the objective measurements of nervous conduction. At week 4 after alcohol exposure (immediately after rhEPO treatment), mice in all groups exhibited evident SSEP waves in response to forepaw stimulation (Fig. 1I). Furthermore, alcohol-exposed mice with no drug treatment in the CAE group showed an obvious decrease in amplitude of forepaw-evoked potential $(0.85 \pm 0.04 \mathrm{mV})$ when compared with those in the Control group (Control: $2.52 \pm 0.14 \mathrm{mV}$; CAE vs. Control, $\mathrm{P}<0.001$; Fig. $1 \mathrm{G}$ and I), indicative of impaired ascending conduction caused by chronic alcoholism. Whereas intranasal EPO-treated mice presented with notably recovered SSEP amplitude (CAE+rhEPO: $2.24 \pm 0.11 \mathrm{mV} ; \mathrm{CAE}+\mathrm{rhEPO}$ vs. $\mathrm{CAE}, \mathrm{P}<0.001)$ which became comparable to that of control mice (CAE+rhEPO vs. Control, $\mathrm{P}>0.05$ ). Coinciding with the change in amplitude, the SSEP latency of alcohol-exposed non-treated mice $(14.00 \pm 0.45 \mathrm{msec})$ was significantly longer than that of non-alcohol-exposed mice (Control: $8.46 \pm 0.44 \mathrm{msec}$; CAE vs. Control, $\mathrm{P}<0.001$; Fig. $1 \mathrm{H}$ and I), indicating the delayed conduction of nervous impulse. Inconsistent with the results of amplitude analysis, although the conduction velocity of forepaw-evoked action potential was restored following intranasal EPO treatment (CAE+rhEPO: $10.36 \pm 0.50 \mathrm{msec}$; CAE+rhEPO vs. CAE, 

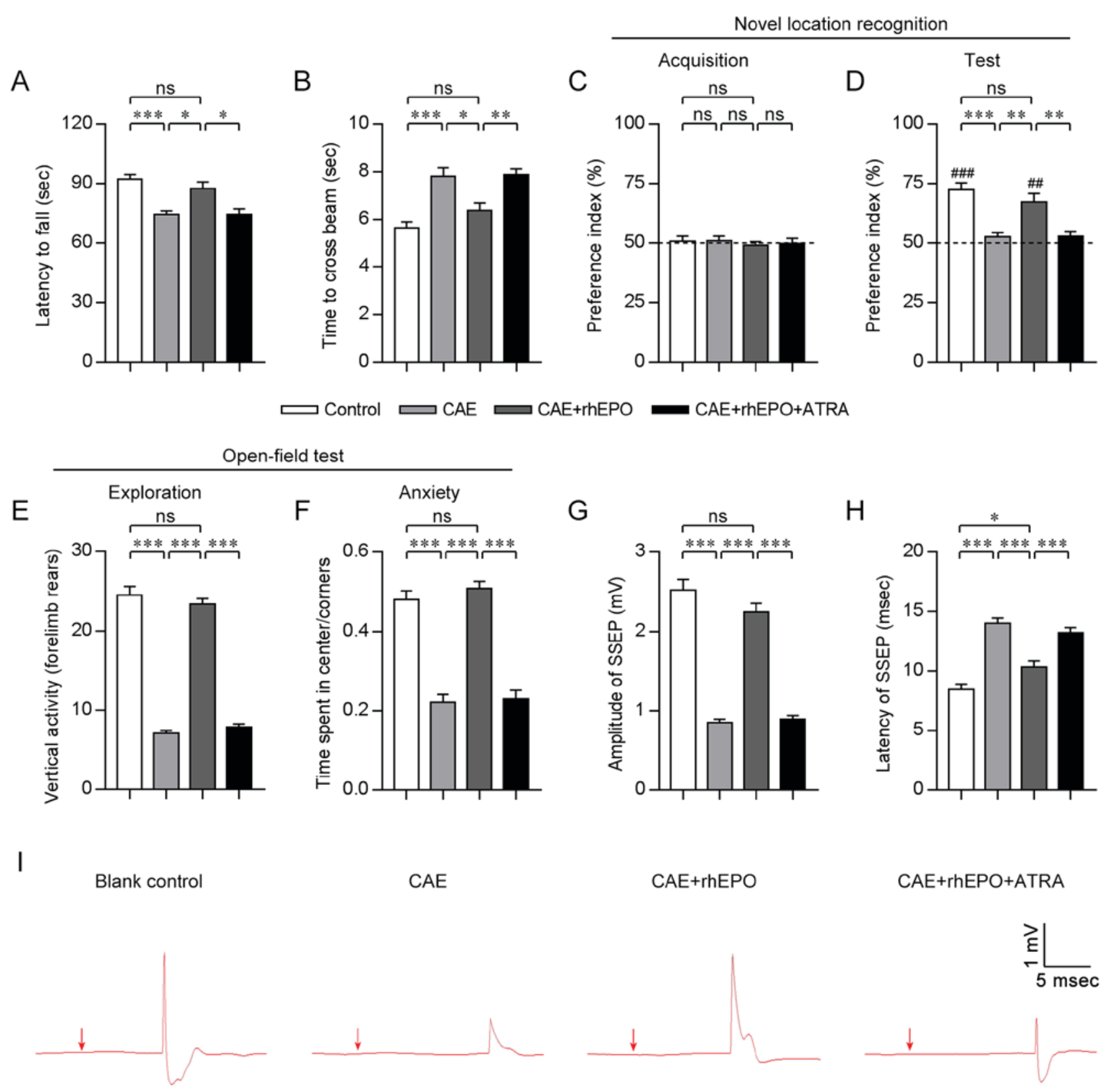

Figure 1. Intranasal rhEPO reverses the motor function deficits, the cognitive disorders and the nervous conduction impairments in mice with chronic alcoholism. (A and B) The quantitative analyses of the latency to fall off rotarod (A) and the time to cross the beam (B) of mice in each group ( $\mathrm{n}=8$ ). (C and D) The quantitative analyses of the preference index of mice in each group on the acquisition (C) and test stages (D) in the novel location recognition test $(n=8)$. $(E$ and $F)$ The quantitative analyses of the vertical activity $(E)$ and the ratio time spent in the center to that in the corner $(F)$ of mice in each group in the open-field test $(n=8)$. ( $\mathrm{G}$ and $\mathrm{H})$ The quantitative analyses of the amplitude $(\mathrm{G})$ and the onset latency $(\mathrm{H})$ of somatosensory-evoked potential (SSEP) of mice in each group $(\mathrm{n}=8)$. (I) The images of SSEP waves of mice in each group. Arrow indicates forepaw stimulation. Calibration bars indicate $1 \mathrm{mV} / 5 \mathrm{msec}$. Error bars indicate SEM. ${ }^{\# \#} \mathrm{P}<0.01$ vs. chance level $50 \%$; ${ }^{\# \# \# ~} \mathrm{P}<0.001$ vs. chance level $50 \%$. ns, non-significant; ${ }^{*} \mathrm{P}<0.05 ;{ }^{* * *} \mathrm{P}<0.01$; ${ }^{* * * *} \mathrm{P}<0.001$. Groups: Control, Chronic Alcohol Exposure (CAE), CAE + recombinant human erythropoietin (rhEPO) and CAE+rhEPO+all-trans-retinoic acid (ATRA).

$\mathrm{P}<0.001)$, the SSEP conduction in EPO-treated mice was still slower than that of control mice $(\mathrm{CAE}+\mathrm{rhEPO}$ vs. Control, $\mathrm{P}<0.05)$. The results of electrophysiological examination verified the therapeutic effects of intranasally administered EPO on the nervous conduction after chronic alcoholism.

Intranasal rhEPO promotes the remyelination in mice with chronic alcoholism. Continuous demyelination and failed remyelination are involved in the prolonged motor coordination impairments and progressive cognitive decline after brain injury (26). As revealed by the immunostaining for MBP (a major constituent of the myelin sheath) performed 4 weeks after alcohol exposure (just after the 4-week rhEPO treatment), the deep layers (V/VI) of the cerebral cortex of mice in the Control group were highly myelinated (Fig. 2A), consistent with a previous description (27). Alcohol-exposed mice in the CAE group showed comparatively impaired myelination in the same cortical layers, which was demonstrated by the decrease in the $\mathrm{MBP}^{+}$myelin sheath $(\mathrm{CAE}$ vs. Control, $\mathrm{P}<0.001$; Fig. 2A and B). When the alcohol-exposed mice were subjected to intranasal EPO treatment, the cortical $\mathrm{MBP}^{+}$myelin level was significantly recovered but did not attain the normal level (CAE+rhEPO vs. CAE, $\mathrm{P}<0.001 ; \mathrm{CAE}+\mathrm{rhEPO}$ vs. Control, 
A
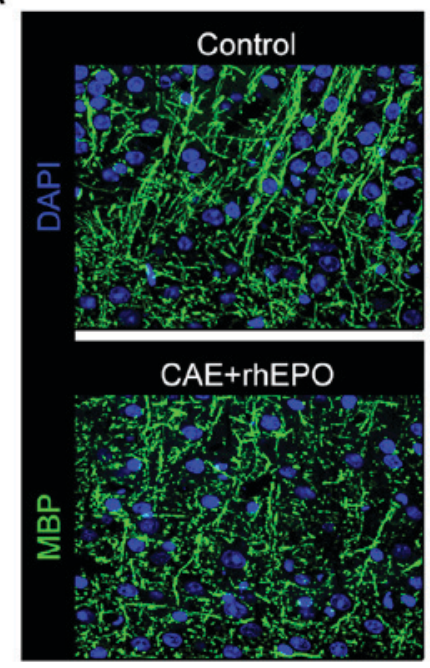

C
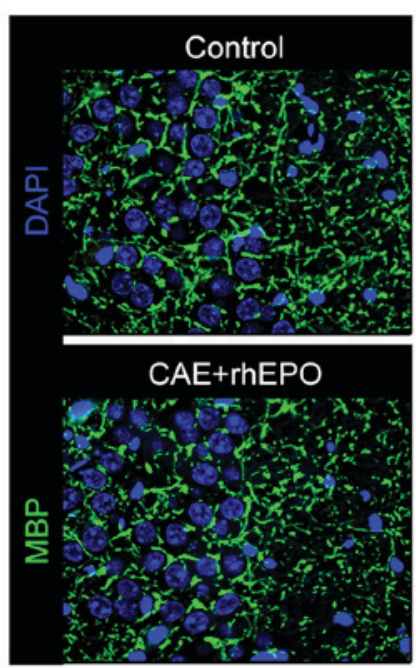

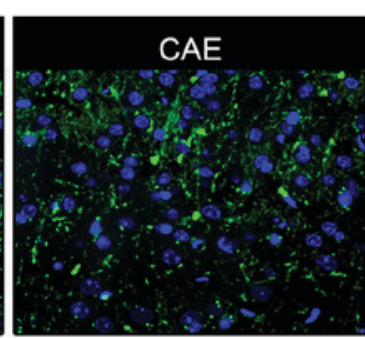

CAE+rhEPO+ATRA
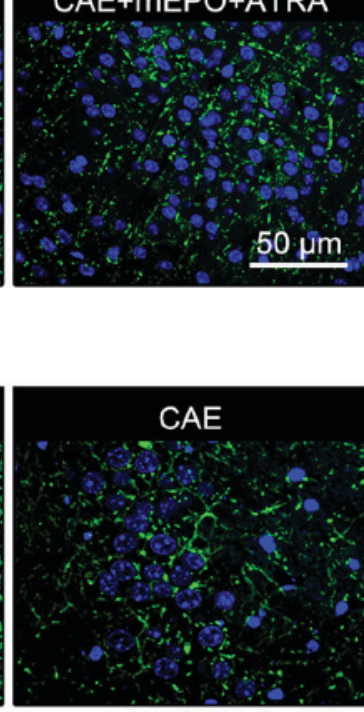

CAE+rhEPO+ATRA

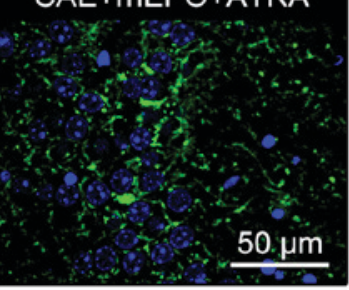

B

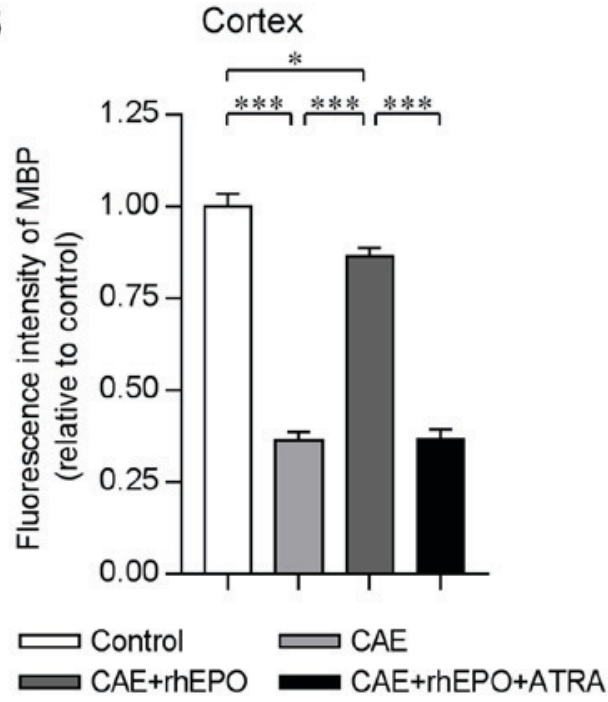

D

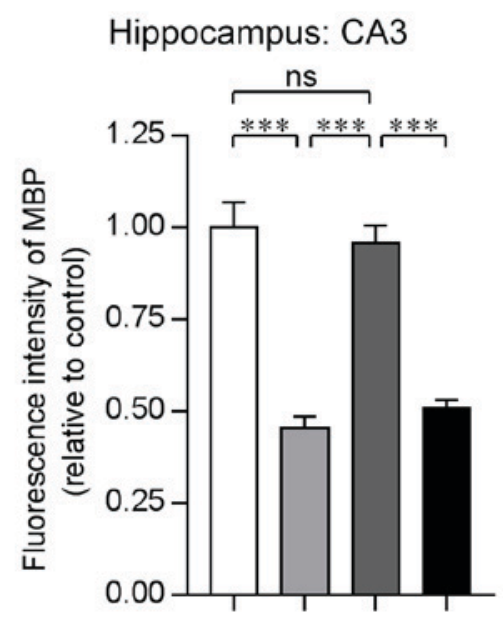

Figure 2. Intranasal rhEPO promotes the remyelination in mice with chronic alcoholism. (A and C) The fluorescence microscopic images of cerebral neocortex (A) and CA3 area of the hippocampus (C) of mice in each group immunostained for myelin basic protein (MBP) and counterstained by DAPI $(n=8)$. (B and D) The quantitative analyses of the relative fluorescence intensity of the cerebral (B) and hippocampal (D) MBP of mice in each group to that in the Control group $(\mathrm{n}=8)$. Scale bar $(\mathrm{A}$ and $\mathrm{C})=50 \mu \mathrm{m}$. Error bars indicate SEM. ns, non-significant; ${ }^{*} \mathrm{P}<0.05 ;{ }^{* * *} \mathrm{P}<0.001 . \mathrm{Groups}$ : Control, Chronic Alcohol Exposure (CAE), CAE + recombinant human erythropoietin (rhEPO) and CAE+rhEPO+all-trans-retinoic acid (ATRA).

$\mathrm{P}<0.05)$. In the CA3 area of the hippocampus rich in myelinated nervous fibers (28), the deteriorated myelination occurred in mice that had been exposed to sustained alcohol (CAE vs. Control, $\mathrm{P}<0.001)$, which was approximately completely reversed in the intranasal EPO-treated mice as determined by stereological volume analysis of MBP fluorescence intensity (CAE+rhEPO vs. CAE, $\mathrm{P}<0.001 ; \mathrm{CAE}+\mathrm{rhEPO}$ vs. Control, $\mathrm{P}>0.05$; Fig. $2 \mathrm{C}$ and D). The data for the immunofluorescence staining for the structural protein of myelin sheath demonstrated the positive effects of intranasal EPO on remyelination in chronic alcoholism-damaged brain.

Intranasal rhEPO accelerates the synapse regeneration in mice with chronic alcoholism. Region-specific synapse loss is closely correlated to interrupted transmission of nervous pulse and impaired cognition in brain injury and neurodegeneration (29-31). To examine the cortical synapse formation which determines cortical synaptic transmission and consequently motor and cognitive function, the immunostaining for synaptophysin (a component of synaptic vesicle) was performed 4 weeks post alcohol exposure ( $12 \mathrm{~h}$ post the end of rhEPO treatment). Quantification of synaptophysin puncta revealed substantial synapse loss in the cortex of the chronic alcohol-exposed mice as compared to their healthy counterpart controls (CAE vs. Control, $\mathrm{P}<0.001$; Fig. 3A and B). After intranasal administration of EPO, the chronic alcohol exposure-affected mice showed significantly elevated fluorescence intensity of synaptophysin in the cortex which was almost equivalent to that of the control mice $(\mathrm{CAE}+\mathrm{rhEPO}$ vs. $\mathrm{CAE}, \mathrm{P}<0.001$; $\mathrm{CAE}+$ rhEPO vs. Control, $\mathrm{P}>0.05)$, suggestive of reconstructed cortical synapse formation supporting the exact nervous conduction and sophisticated neural functions. To further evaluate the cognition-related hippocampal synaptogenesis after alcohol exposure and EPO treatment, TEM analysis was conducted to recognize the ultramicroscopic structures of synapse. As determined by presynaptic (synaptic vesicle) 
A
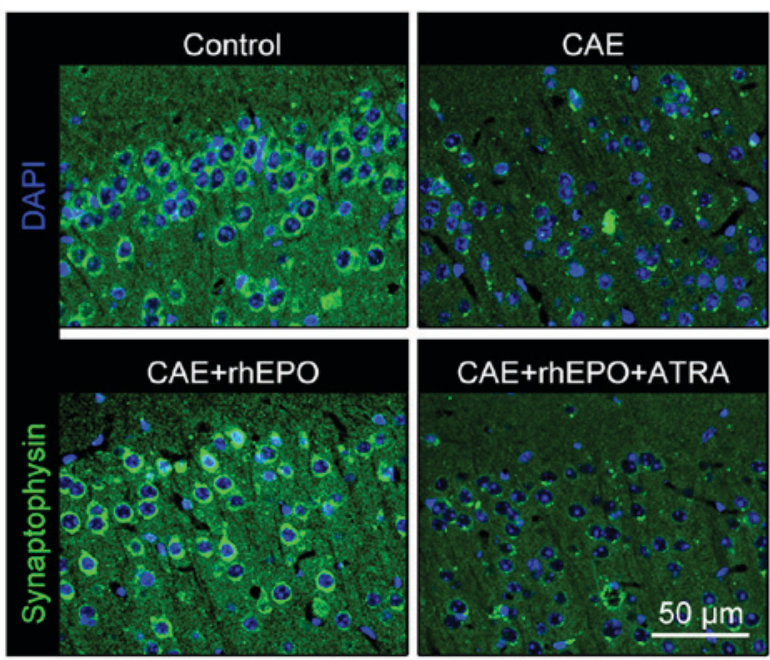

C

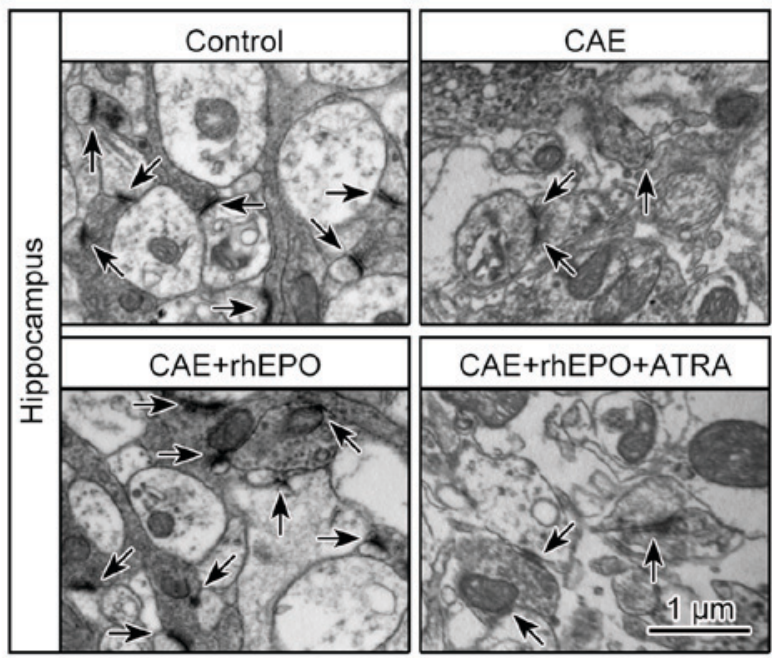

B

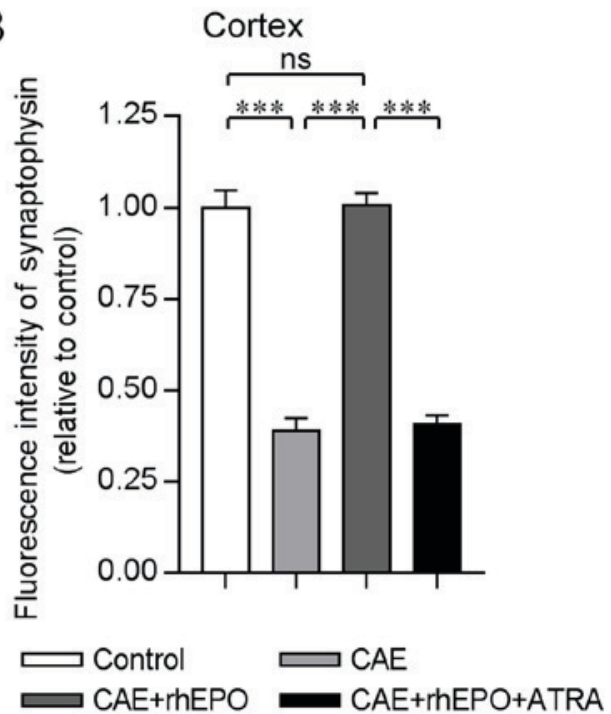

D

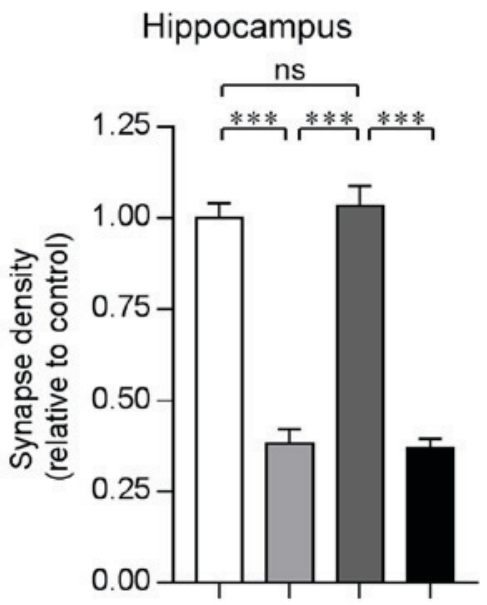

Figure 3. Intranasal rhEPO accelerates the synapse regeneration in mice with chronic alcoholism. (A) The fluorescence microscopic images of the cerebral neocortex of mice in each group immunostained for synaptophysin and counterstained by DAPI. (B) The quantitative analysis of the relative fluorescence intensity of cerebral synaptophysin of mice in each group to that in the Control group (n=8). (C) The ultramicroscopic (TEM) images of the hippocampus of mice in each group. (D) The quantitative analysis of the hippocampal synapse density of mice in each group to that in the Control group (n=8). Scale bar $(\mathrm{A})=50 \mu \mathrm{m} ;(\mathrm{C})=1 \mu \mathrm{m}$. Error bars indicate SEM. ns, non-significant; ${ }^{* * *} \mathrm{P}<0.001$. Groups: Control, Chronic Alcohol Exposure $(\mathrm{CAE})$, CAE + recombinant human erythropoietin (rhEPO) and CAE+rhEPO+all-trans-retinoic acid (ATRA).

and postsynaptic compartments (postsynaptic density) in electron micrographs, hippocampal synapse density obviously declined on prolonged alcohol exposure in mice of the CAE group in contrast to that in controls (CAE vs. Control, $\mathrm{P}<0.001$; Fig. 3C and D), but synapse amount recovered on intranasal EPO treatment and reached the non-alcohol-affected level in the CAE+rhEPO group (CAE+rhEPO vs. CAE, $\mathrm{P}<0.001$ $\mathrm{CAE}+\mathrm{rhEPO}$ vs. Control, $\mathrm{P}>0.05)$, which was consistent with the tendency obverse in cortex. These data clarified the repairing potential of intranasal EPO for brain synaptogenesis disrupted in chronic alcoholism.

Intranasal rhEPO exerts neuroprotective effects on mice with chronic alcoholism by regulating the autophagy-related degradation of Nrf2. To establish the mechanisms underlying the neuroprotective effects of intranasal rhEPO, western blot analyses were performed on the microdissected cortex and hippocampus $30 \mathrm{~min}$ after EPO administration for the last time. As expected, hEPO was detected in the brain tissue of intranasal EPO-treated mice rather than non-treated mice (Fig. 4A). Correspondingly, the exogenous EPO caused a considerable increase in phosphorylated (p)-EPOR, p-ERK1/2 and p-AKT levels in the cerebrum of the treated mice (CAE+rhEPO vs. CAE, all P-values <0.001; CAE+rhEPO vs. Control, P-values <0.001; Fig. 4A-D), reminiscent of the augmented activation of EPOR, ERKs and PI3K/AKT signaling. Furthermore, intranasally delivered EPO led to the upregulated level of cerebral antioxidant and neuroprotective Nrf2, in parallel with the reduced level of Nrf2 inhibitor protein KEAP1 and the elevated protein expression of Nrf2 target gene NQO1 (CAE+rhEPO vs. CAE, all P-values $<0.001$; Fig. 4A and E-G), which suggested the increased Nrf2 activity by EPO. However, mere chronic alcohol exposure resulted in a detectable reduction in basal EPOR, ERK1/2, AKT and Nrf2 activity (CAE vs. Control, all P-values <0.05). 

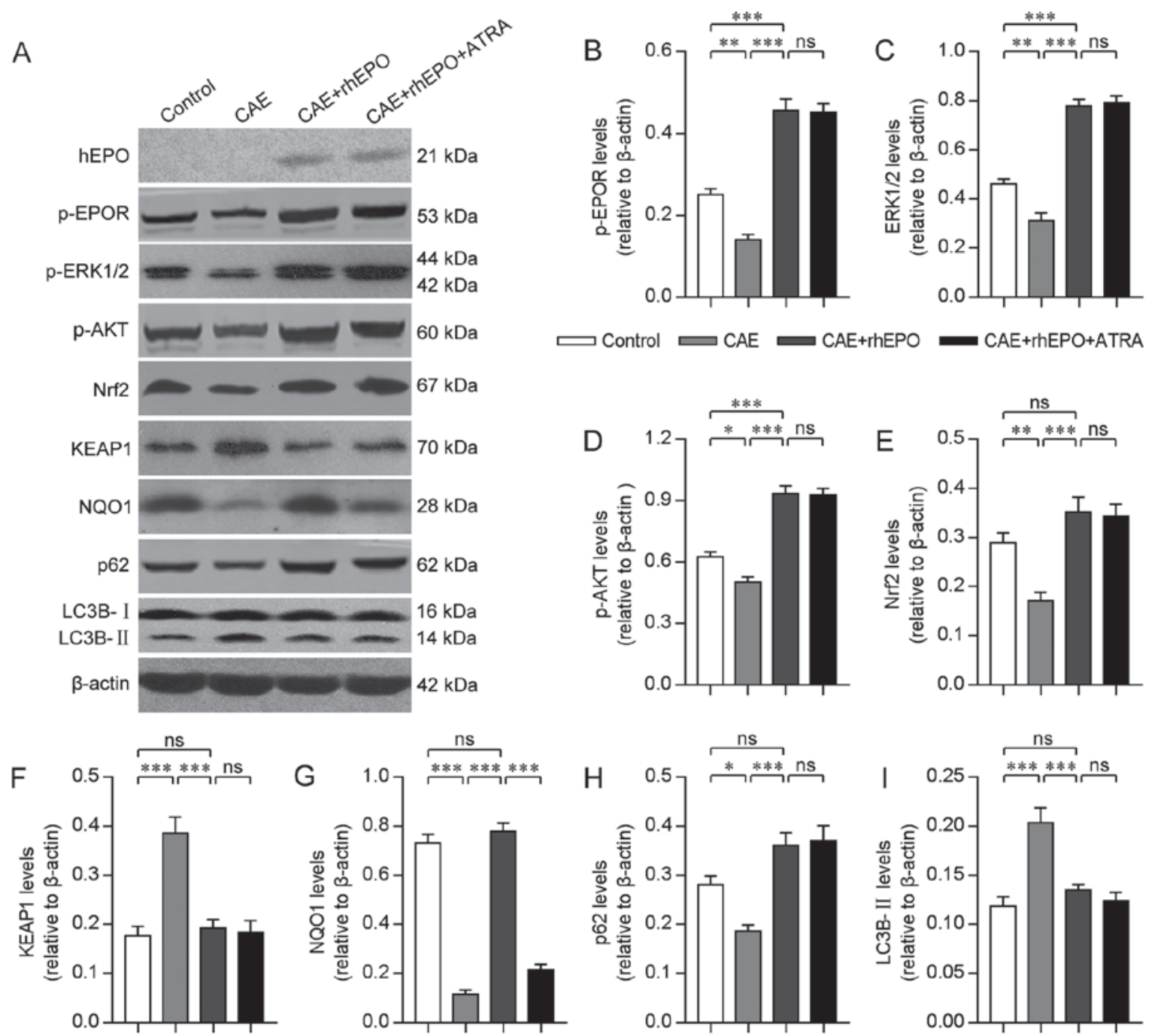

Figure 4. Intranasal rhEPO exerts neuroprotective effects by regulating autophagy-related Nrf2 degradation in mice with chronic alcoholism. (A) Images of western blot analyses for hEPO, phosphorylated (p)-EPOR, p-ERK1/2, p-AKT, Nrf2, KEAP1, NQO1, p62 and LC3-II in brain tissue of mice in each group. (B-I) The quantitative analyses of the relative p-EPOR (B), p-ERK1/2 (C), p-AKT (D), Nrf2 (E), KEAP1 (F), NQO1 (G), p62 (H) and LC3-II (I) levels to $\beta$-actin in brain tissue of mice in each group $(\mathrm{n}=8)$. Error bars indicate SEM. $\mathrm{ns}$, non-significant; ${ }^{*} \mathrm{P}<0.05 ;{ }^{* * *} \mathrm{P}<0.01$; ${ }^{* * *} \mathrm{P}<0.001$. Groups: Control, Chronic Alcohol Exposure (CAE), CAE + recombinant human erythropoietin (rhEPO) and CAE+rhEPO+all-trans-retinoic acid (ATRA).

As autophagy-degraded p62 is reported to enhance Nrf2 signaling by inactivating KEAP1 $(15,17)$, the relationship between Nrf2 activity and autophagy progress was monitored. The accelerated degradation of p62 by autophagy and the autophagy-indicative accumulation of LC3-II in alcohol-affected brain tissue (CAE vs. Control, both P-values $<0.05$; Fig. 4A, $\mathrm{H}$ and I) was reversed by intranasal rhEPO (CAE+rhEPO vs. CAE, all P-values $<0.001$ ), coinciding with the EPO-activated autophagy-inhibitory ERKs and PI3K/AKT signaling, the reduced level of Nrf2-inhibiting KEAP1, and the rescued expression of Nrf2 and Nrf2-targeted NQO1. These observations indicated the involvement of antioxidant Nrf2 negatively regulated by the alcohol-induced autophagic response in the protective impacts of EPO.

To further determine whether EPO functions as neuroprotector by modulating Nrf2 activity, ATRA, the specific antagonist of Nrf2 $(16,32)$, was administered intraperitoneally 30 min before intranasal EPO treatment for each time. The effective suppression of Nrf2 activity by ATRA was demonstrated by the downregulated protein level of NQO1
(CAE+rhEPO+ATRA vs. CAE+rhEPO, both P-values <0.001; Fig. 4A and G). Expectedly, the levels of intracerebral hEPO, activated EPOR and regulatory molecules (ERKs and AKT), autophagy-related proteins (p62 and LC3-II), KEAP1 and also Nrf2 were not influenced by ATRA-induced Nrf2 inhibition $(\mathrm{CAE}+\mathrm{rhEPO}+\mathrm{ATRA}$ vs. CAE+rhEPO, all P-values $>0.05$; Fig. 4A-C and F-H). However, if pre-treated by ATRA, the intranasal EPO-administered mice with chronic alcohol exposure showed significantly curtailed duration maintaining on the rotarod (Fig. 1A), delayed beam-crossing (Fig. 1B), abolished preference for displaced object in novel location recognition task (Fig. 1D), impaired exploration-learning activity and increased anxiety in open-field test (Fig. 1E and F), retarded conduction of SSEP with amplitude loss (Fig. 1G-I), decreased $\mathrm{MBP}^{+}$myelin sheath in cerebral cortex (Fig. 2A and B) and hippocampus (Fig. 2C and D), diminished cortical synaptophysin level (Fig. 3A and B) and reduced hippocampal synapse density (Fig. 3C and D) (CAE+rhEPO+ATRA vs. CAE+rhEPO, all P-values $<0.05$ ), which suggested that the EPO-induced recovery in motor cooperation, cognition, 
nervous conduction and cerebral myelination and synaptogenesis were almost abrogated by the inhibition of Nrf2 activity. These data confirmed that the therapeutic effects of intranasal EPO on chronic alcoholism-related brain disorders were mediated, at least partially, by impeding the autophagy-related degradation of $\mathrm{Nrf} 2$.

\section{Discussion}

In the present study, we discovered the therapeutic effects of intranasally administered erythropoietin (EPO) on the neurological function deficits and neural pathology in chronic alcoholism, and identified Nrf2 activity as the central mechanism underlying the neuroprotection. EPO, a well-established hematopoietic factor, was recently detected in the central nervous system (CNS) where it acts as the trophic and protective factor for neurons as well as glia $(7,33)$. Dysfunction of EPO-EPOR signaling is implicated in the pathogenesis of neurogenerative diseases (such as amyotrophic lateral sclerosis), and treatment with exogenous EPO has been reported to rescue the functional defects and pathological alterations in the neurodegeneration and traumatic and ischemic injuries of CNS (7-12). However, the effects of EPO on chronic alcoholism-related brain damages were poorly understood previously.

To evaluate the therapeutic potential of EPO for the neurological disorders and pathology, mice were subjected to CIE vapor inhalation in order to recapitulate the neurological function defects in human chronic alcoholism and the correlated neuropathology and pathogenesis. This vapor inhalation procedure is a canonical mouse model of chronic alcoholism and widely used in the researches on the neural mechanisms underlying the chronic alcohol exposure-induced behavioral and cognitive alterations (24). In the present study, chronic alcohol-exposed mice displayed obviously impaired motor cooperation and velocity in rotarod and beam walk tests, reduced discriminative memory for the displayed object in novel location recognition task, and decreased exploration activity and exacerbated anxiety in open field (Fig. 1A-F), reminiscent of the clinical manifestations of motor and cognitive dysfunction in patients with chronic alcoholism (3). In the present study, rhEPO was administered via noninvasive intranasal route, which allows rapid delivery of therapeutic extrinsic cytokines and extracellular vesicles directly to the brain from the nasal mucosa, bypasses the blood-brain barrier that limits the distribution of systemically administered therapeutics to the CNS, and minimizes systemic exposure $(25,34)$. Our findings further supported the feasibility of intranasal administration of EPO, as evidenced by the entry of exogenous rhEPO into mouse brain tissue and the consequent activation of EPOR and the downstream ERKs and PI3K/AKT signaling (Fig. 4A-C). After treatment with EPO delivered via intranasal route, alcohol-exposed animals gained the approximately normal complex body movement and coordination, significantly reversed spatial reference and work memory, and notably restored exploration and learning drive and alleviated anxiety status, which verified the therapeutic efficacy of EPO for the sustained alcohol exposure-caused locomotor, cognitive and emotional disorders in mice. Furthermore, somatosensory-evoked potential (SSEP) was exploited to objectively measure the nervous conduction of CNS as reported previously (29). Similar to the observations in the behavioral tests, intranasal EPO led to notable recovery from the prolonged alcohol exposure-induced amplitude loss and conduction delay of SSEP, albeit not full restoration in the onset latency (Fig. 1G-I).

Normal myelination is known to be essential for the normal function of the CNS. Abnormal myelination in neurodevelopmental defects, and also demyelination and failed remyelination in brain injury are correlated to impaired transmission of nervous pulse as well as the metabolic disturbance and dysfunction of neural axon, which consequentially affects motor and cognitive functions (26). Our findings indicated that chronic alcohol exposure brought about poor myelination in the deep layers (V/VI) of the cerebral cortex and the CA3 area of hippocampus in mice, where nervous fibers are highly myelinated as observed previously $(27,28)$. The repair potential of intranasal EPO for the alcohol-induced myelin damage was demonstrated by the reversion of the MBP-positive myelin sheath in the cortex and hippocampus to the uninjured levels (Fig. 2), associated with the recovery of affected nervous conduction, locomotor and cognition. Synapse formation is another major determinant for transmission of nervous signala, and complicatedly regulated motor and cognitive activity. Disrupted synaptogenesis is established to be the common mechanism mediating the neurological disorders of neurodegeneration and damages in CNS $(30,31)$. In the present study, sustained alcohol generated marked synaptic toxicity in the affected cortex and hippocampus, as revealed by the decreased level of cortical synaptophysin (a canonical marker of synapse) and the reduced hippocampal synapse density (Fig. 3). Following intranasal treatment with rhEPO, the EPO-provoked regeneration of synapse was observed in the cortex, accounting for the restored cortical synaptic transmission reflected by forepaw-evoked potential (29). The synaptogenesis-promoting capacity of EPO was also detected in the hippocampus, which could be related to the improved behaviors of treated mice in hippocampus-dependent cognitive tasks. Therefore, our data indicated that intranasally delivered EPO conferred profound neuroprotection in the brain against sustained alcohol exposure. Of note, the incompletely restored SSEP conduction suggested that the newly regenerated myelin and synapse immediately after EPO treatment might be immature in structure and function, which should be further studied in the future.

Nrf 2 acts as the major regulator of cellular resistance to oxidant stress and also chemical toxicity associated with oxidative pathology by controlling the basal and induced expression of an array of antioxidant response element-dependent genes (i.e., NQO1 and HO-1) (14,15). Recently, pharmacological boosting of Nrf2 activity with EPO is reported to induce protection against neurotoxin- and aging-related neural damages $(18,20)$. In this study, we observed that the level of Nrf2 and the expression of Nrf2 target gene NQO1 were elevated by extrinsic EPO in the brain tissue of alcohol-exposed mice (Fig. 4). Consistently, the preservation of motor cooperation, cognition, nervous conduction, myelination and synaptogenesis by EPO was almost abolished upon pre-treatment with ATRA (Figs. 1-3), the well-established blood-brain barrier-permeable potent antagonist of $\mathrm{Nrf} 2(16,32)$, further demonstrating that 
Nrf2 signaling mediated the neuroprotective effects of EPO in chronic alcoholism. As Nrf2 mRNA is expressed constitutively and independently of inducers, the protein turnover via KEAP1-ubiquitin-proteasome pathway is suggested as the major mechanism regulating Nrf2 activation (14). Interestingly, p62, a substrate for autophagic degradation, can augment Nrf2 activity by recruiting and inactivating KEAP1 in embryonic neurodevelopment and the pharmacologically induced macrophagic tolerance to inflammatory stimuli, indicative of the negative correlation between $\mathrm{Nrf} 2$ activity and autophagic reaction $(15,17)$. In mice with chronic alcoholism-like pathology, we detected that intranasal rhEPO suppressed alcohol-provoked cerebral autophagic response by stimulating ERKs and PI3K/AKT signaling, as do other growth and neurotrophic factors $(35,36)$. Simultaneously, the rescued expression of p62 was closely related to the degradation of Nrf2-sequestering KEAP1 as well as the restored levels of Nrf2 and Nrf2-targeted NQO1. Upon the inhibition of Nrf2 activity by ATRA, the NQO1 expression was significantly reduced, whereas the level of intracerebral hEPO, the expression of signaling molecules upstream of Nrf2 (the EPOR signaling as well as autophagic progression-related proteins), the level of KEAP1 regulating the accumulation of Nrf2, and even the expression of Nrf2, were expectedly unaffected (Fig. 4), which was consistent with the highly specific antagonism of ATRA against Nrf2 (32). Our data suggested that EPO could activate the neuroprotective Nrf2 activity in chronic alcohol-affected brain tissue by hindering the autophagy-related degradation of Nrf2 via ERKs and PI3K/AKT signaling. This previously unanticipated mechanism may also underlie the EPO-conferred neuroprotection against other neurotoxins and aging.

In summary, we found that intranasal EPO effectively rescued motor dysfunction, cognitive and emotional disorders, and nervous conduction impairment caused by sustained alcohol exposure in this study. Consistently, the intranasally delivered EPO promoted the remyelination and synapse formation in chronic alcoholism-affected neocortex and hippocampus. Moreover, we discovered that the exogenous rhEPO, which entered the cerebrum through the intranasal route, activated EPOR and downstream ERKs and PI3K/AKT signaling, and suppressed autophagy-related degradation of Nrf2. We further presented evidence to show that Nrf2 activity mediated the intranasal EPO-exerted neuroprotection.

\section{Acknowledgements}

The authors would like to thank Dr Caichuan Yan from Heilongjiang Institute of Cancer Prevention and Treatment for providing technical support.

\section{Funding}

The present study was supported by a research award from the Health and Family Planning Commission of Heilongjiang Province (2016-041).

\section{Availability of data and materials}

All data generated or analyzed during this study are included in this published article.

\section{Authors' contributions}

HS designed the study, performed the experiments and modified the manuscript. XN performed the CIE vapor inhalation procedure and the related treatments, conducted the immunofluorescence staining, analyzed the data, and was a major contributor in writing the manuscript. WW performed the TEM and western blotting analyses and analyzed the data. QW conducted the motor and cognitive behavioral tests and analyzed the data. DZ performed the nerve conduction tests and analyzed the data. All authors helped to draft, read and approved the final manuscript.

\section{Ethics approval and consent to participate}

The manuscript involves no patients and human samples. All experimental procedures on animals were approved by the Ethics Committee of Harbin Medical University and the Ethics Committee of Qiqihar Medical University, and followed the principles of medical ethics.

\section{Patient consent for publication}

Not applicable.

\section{Competing interests}

The authors declare that they have no competing interests.

\section{References}

1. Ron D and Barak S: Molecular mechanisms underlying alcohol-drinking behaviours. Nat Rev Neurosci 17: 576-591, 2016.

2. Fama R and Sullivan EV: Methods of association and dissociation for establishing selective brain-behavior relations. Handb Clin Neurol 125: 175-181, 2014.

3. Lembke A and Stanford M: Clinical management of alcohol use disorders in the neurology clinic. Handb Clin Neurol 125: 659-670, 2014.

4. Gupta S and Warner J: Alcohol-related dementia: A 21st-century silent epidemic? Br J Psychiatry 193: 351-353, 2008.

5. Vetreno RP and Crews FT: Current hypotheses on the mechanisms of alcoholism. Handb Clin Neurol 125: 477-497, 2014.

6. Connor JP, Haber PS and Hall WD: Alcohol use disorders. Lancet 387: 988-998, 2016.

7. Sato K, Morimoto N, Kurata T, Mimoto T, Miyazaki K, Ikeda Y and Abe K: Impaired response of hypoxic sensor protein HIF-1 $\alpha$ and its downstream proteins in the spinal motor neurons of ALS model mice. Brain Res 1473: 55-62, 2012.

8. Koh S, Kim Y, Kim HY, Cho GW, Kim KS and Kim SH: Recombinant human erythropoietin suppresses symptom onset and progression of G93A-SOD1 mouse model of ALS by preventing motor neuron death and inflammation. Eur J Neurosci 25: 1923-1930, 2007.

9. Rodriguez Cruz Y, Strehaiano M, Rodriguez Obaya T, Garcia Rodriguez JC and Maurice T: An intranasal formulation of erythropoietin (Neuro-EPO) prevents memory deficits and amyloid toxicity in the APPSwe transgenic mouse model of Alzheimer's disease. J Alzheimers Dis 55: 231-248, 2017.

10. Cerri G, Montagna M, Madaschi L, Merli D, Borroni P, Baldissera $\mathrm{F}$ and Gorio A: Erythropoietin effect on sensorimotor recovery after contusive spinal cord injury: An electrophysiological study in rats. Neuroscience 219: 290-301, 2012.

11. Hellewell SC, Yan EB, Alwis DS, Bye $N$ and Morganti-Kossmann MC: Erythropoietin improves motor and cognitive deficit, axonal pathology, and neuroinflammation in a combined model of diffuse traumatic brain injury and hypoxia, in association with upregulation of the erythropoietin receptor. J Neuroinflammation 10: 156, 2013. 
12. Ma S, Chen J, Chen C, Wei N, Xu J, Yang G, Wang N, Meng Y, Ren $\mathrm{J}$ and $\mathrm{Xu} Z$ : Erythropoietin rescues memory impairment in a rat model of chronic cerebral hypoperfusion via the EPO-R/JAK2/STAT5/PI3K/Akt/GSK-3 $\beta$ pathway. Mol Neurobiol 55: 3290-3299, 2018.

13. Chong ZZ, Shang YC, Mu Y, Cui S, Yao Q and Maiese K: Targeting erythropoietin for chronic neurodegenerative diseases. Expert Opin Ther Targets 17: 707-720, 2013

14. Ma Q: Role of nrf2 in oxidative stress and toxicity. Annu Rev Pharmacol Toxicol 53: 401-426, 2013.

15. Jang J, Wang Y, Lalli MA, Guzman E, Godshalk SE, Zhou H and Kosik KS: Primary cilium-autophagy-Nrf2 (PAN) axis activation commits human embryonic stem cells to a neuroectoderm fate. Cell 165: 410-420, 2016

16. Yin XP, Zhou J, Wu D, Chen ZY and Bao B: Effects of that ATRA inhibits Nrf2-ARE pathway on glial cells activation after intracerebral hemorrhage. Int J Clin Exp Pathol 8: 10436-10443, 2015.

17. Mildenberger J, Johansson I, Sergin I, Kjobli E, Damas JK, Razani B, Flo TH and Bjorkoy G: N-3 PUFAs induce inflammatory tolerance by formation of KEAP1-containing SQSTM1/p62-bodies and activation of NFE2L2. Autophagy 13: 1664-1678, 2017.

18. Shen J, Wu Y, Xu J, Zhang J, Sinclair SH, Yanoff M, Xu G, Li W and Xu G: ERK- and Akt-dependent neuroprotection by erythropoietin (EPO) against glyoxal-AGEs via modulation of Bcl-xL, Bax, and BAD. Invest Ophthalmol Vis Sci 51: 35-46, 2010.

19. Genc K, Egrilmez MY and Genc S: Erythropoietin induces nuclear translocation of Nrf2 and heme oxygenase-1 expression in SH-SY5Y cells. Cell Biochem Funct 28: 197-201, 2010.

20. Wu H, Zhao J, Chen M, Wang H, Yao Q, Fan J and Zhang M: The anti-aging effect of erythropoietin via the ERK/Nrf2-ARE pathway in aging rats. J Mol Neurosci 61: 449-458, 2017.

21. Schattenberg JM and Czaja MJ: Regulation of the effects of CYP2E1-induced oxidative stress by JNK signaling. Redox Biol 3: 7-15, 2014

22. Dekeyser GJ, Clary CR and Otis JS: Chronic alcohol ingestion delays skeletal muscle regeneration following injury. Regen Med Res 1: 2, 2013.

23. Roman J: Chronic alcohol ingestion and predisposition to lung 'cirrhosis'. Alcohol Clin Exp Res 38: 312-315, 2014.

24. Holmes A, Fitzgerald PJ, Macpherson KP, Debrouse L, Colacicco G, Flynn SM, Masneuf S, Pleil KE, Li C, Marcinkiewcz CA, et al: Chronic alcohol remodels prefrontal neurons and disrupts NMDAR-mediated fear extinction encoding. Nat Neurosci 15: 1359-1361, 2012

25. Scafidi J,Hammond TR, Scafidi S, Ritter J, Jablonska B, Roncal M, Szigeti-Buck K, Coman D, Huang Y, McCarter RJ Jr, et al: Intranasal epidermal growth factor treatment rescues neonatal brain injury. Nature 506: 230-234, 2014.
26. Piao J, Major T, Auyeung G, Policarpio E, Menon J, Droms L, Gutin P, Uryu K, Tchieu J, Soulet D and Tabar V: Human embryonic stem cell-derived oligodendrocyte progenitors remyelinate the brain and rescue behavioral deficits following radiation. Cell Stem Cell 16: 198-210, 2015.

27. Tomassy GS, Berger DR, Chen H, Kasthuri N, Hayworth KJ, Vercelli A, Seung HS, Lichtman JW and Arlotta P: Distinct profiles of myelin distribution along single axons of pyramidal neurons in the neocortex. Science 344: 319-324, 2014

28. Ahn JH, Lee TK, Park JH, Cho JH, Kim IH, Lee JC, Hong S, Jeon YH, Kang IJ, Lee YJ, et al: Age-dependent differences in myelin basic protein expression in the hippocampus of young, adult and aged gerbils. Lab Anim Res 33: 237-243, 2017.

29. Chen S, Mohajerani MH, Xie Y and Murphy TH: Optogenetic analysis of neuronal excitability during global ischemia reveals selective deficits in sensory processing following reperfusion in mouse cortex. J Neurosci 32: 13510-13519, 2012.

30. Perez EJ, Tapanes SA, Loris ZB, Balu DT, Sick TJ, Coyle JT and Liebl DJ: Enhanced astrocytic d-serine underlies synaptic damage after traumatic brain injury. J Clin Invest 127: 3114-3125, 2017.

31. Hong S, Bejaglasser VF, Nfonoyim BM, Frouin A, Li S, Ramakrishnan S, Merry K, Shi Q, Rosenthal A, Barres BA, et al: Complement and microglia mediate early synapse loss in Alzheimer mouse models. Science 352: 712-716, 2016.

32. Meng QT, Cao C, Wu Y, Liu HM, Li W, Sun Q, Chen R, Xiao YG, Tang LH, Jiang Y, et al: Ischemic post-conditioning attenuates acute lung injury induced by intestinal ischemia-reperfusion in mice: Role of Nrf2. Lab Invest 96: 1087-1104, 2016.

33. Dale EA, Satriotomo I and Mitchell GS: Cervical spinal erythropoietin induces phrenic motor facilitation via extracellular signal-regulated protein kinase and Akt signaling. J Neurosci 32: 5973-5983, 2012

34. Dhuria SV, Hanson LR and Frey WH II: Intranasal delivery to the central nervous system: Mechanisms and experimental considerations. J Pharm Sci 99: 1654-1673, 2010.

35. Noda NN and Inagaki F: Mechanisms of autophagy. Annu Rev Biophys 44: 101-122, 2015.

36. Galluzzi L, Bravo-San Pedro JM, Blomgren K and Kroemer G: Autophagy in acute brain injury. Nat Rev Neurosci 17: 467-484, 2016.

This work is licensed under a Creative Commons Attribution-NonCommercial-NoDerivatives 4.0 International (CC BY-NC-ND 4.0) License. 